\title{
Hydrophobic Fluorous Metal-Organic Framework Nanoadsorbent for Removal of Hazardous Wastes from Water
}

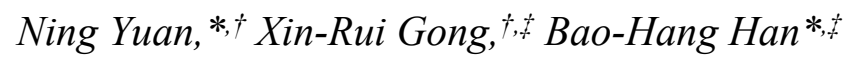 \\ $\uparrow$ School of Chemical and Environmental Engineering, China University of Mining and Technology, \\ Beijing 100083, China \\ \$ CAS Key Laboratory of Nanosystem and Hierarchical Fabrication, CAS Center for Excellence in \\ Nanoscience, National Center for Nanoscience and Technology, Beijing 100190, China.
}

E-mail: ning.yuan@hotmail.com

E-mail: hanbh@nanoctr.cn 


\section{Materials}

Zirconium tetrachloride $\left(\mathrm{ZrCl}_{4}\right)$, pentadecafluorooctanoyl chloride, carbamazepine, and sodium tungstate dihydrate $\left(\mathrm{Na}_{2} \mathrm{WO}_{4}\right)$ were purchased from Aladdin Industrial Corporation. 2-Aminoterephthalic acid ( $\mathrm{H}_{2} \mathrm{ATA}$ ) was supplied from J\&K Scientific Ltd. Chloroform, dichloromethane, toluene, heptane, $n$ hexane, silicone oil, methanol $(\mathrm{MeOH})$, ethanol $(\mathrm{EtOH})$, acetone, petroleum ether, $N, \quad N-$ dimethylformamide (DMF), and formic acid were obtained from Beijing Chemical Factory. Sodium acetate $\left(\mathrm{CH}_{3} \mathrm{COONa}\right)$, sodium carbonate $\left(\mathrm{Na}_{2} \mathrm{CO}_{3}\right)$, and sodium sulfate $\left(\mathrm{Na}_{2} \mathrm{SO}_{4}\right)$ were obtained from Shanghai Macklin Biochemical Co., Ltd. Sodium chloride $(\mathrm{NaCl})$ was obtained from Xilong Scientific Co., Ltd.

\section{Instrumental Characterization}

The ${ }^{1} \mathrm{H}$ NMR spectra of both MOFs and organic compounds were recorded on a Bruker Advance III 400 NMR spectrometer (Bruker Daltonics Inc., Germany). The MOFs were digested with a solution of CsF-D 2 O-DMSO- $d_{6}$. FTIR spectra were obtained by using a Spectrum One FTIR spectroscope (Perkin Elmer Instrument, USA). TGA was performed at a Diamond TG/DTA thermogravimetric differential thermal synchronous thermal analyzer (Perkin Elmer, USA). Thermogravimetric analysis was performed in an air and nitrogen atmosphere on a $10^{\circ} \mathrm{C} \mathrm{min}^{-1}$ ramp from room temperature to $800{ }^{\circ} \mathrm{C}$. Contact angles were measured using DSA-100 (KRÜSS GmbH, Germany) by the sessile-drop method. The composition of the samples was studied by PXRD in a D/MAX-TTRIII(CBO) diffractometer (Rigku, Japan). SEM images of samples were obtained with GeminiSEM 300 (ZEISS, Germany). The TEM images were recorded on JEM 1200EX (JEOL, Japan) transmission electron microscope. The zeta potential was obtained with a Zetasizer Nano S Nanoscale potentiometer (Malvern, UK). The X-ray photoelectron spectra (XPS) were obtained on a Thermo ESCALAB 250XI instrument (Thermo Scientific, USA). The UV-Vis spectra were collected by a Lambda 950 UV-Vis-NIR spectrometer (Perkin Elmer, USA). The 
nitrogen adsorption-desorption isotherms were obtained by the Autosorb IQ surface area and porosity analyzer (Quantachrome Instruments, USA) at $77 \mathrm{~K}$.

\section{Synthesis of UiO-66- $\mathrm{NH}_{2}$}

Typically, a mixture of $0.17 \mathrm{~g} \mathrm{ZrCl}_{4}$ and $0.13 \mathrm{~g}$ 2-aminoterephthalic acid was added in $8 \mathrm{~mL} \mathrm{DMF}$. After the mixture was dissolved, $0.8 \mathrm{~mL}$ of formic acid was added. The solution was put in the autoclave. The sealed autoclave was heated at $120^{\circ} \mathrm{C}$ in an air-circulating oven for $24 \mathrm{~h}$. After cooling down, the obtained material was collected via centrifugation and washed with DMF and acetone. It was then Soxhlet extracted in acetone for $48 \mathrm{~h}$ to remove DMF. Ultimately, the powder was dried at $80{ }^{\circ} \mathrm{C}$ for $3 \mathrm{~h}$.

\section{Synthesis of $\mathrm{BDC}-\mathrm{C}_{7} \mathrm{~F}_{15}$}

BDC- $\mathrm{C}_{7} \mathrm{~F}_{15}$ was prepared according to the previously reported method. ${ }^{\mathrm{S} 1}$ In an ice water bath, 2aminoterephthalic acid $(2.0 \mathrm{~g}, 11.05 \mathrm{mmol})$ and $15 \mathrm{~mL}$ DMF were added to a $50 \mathrm{~mL}$ round bottom flask. After it was completely dissolved, $2.8 \mathrm{~mL}$ perfluorooctanoyl chloride was added. Then, $12.3 \mathrm{mmol}$ triethylamine solution was added dropwise within $30 \mathrm{~min}$, and the mixture was stirred at room temperature for $1 \mathrm{~h}$. Afterward, the mixed solution was poured into ice water, filtered, washed with water, and dried. 


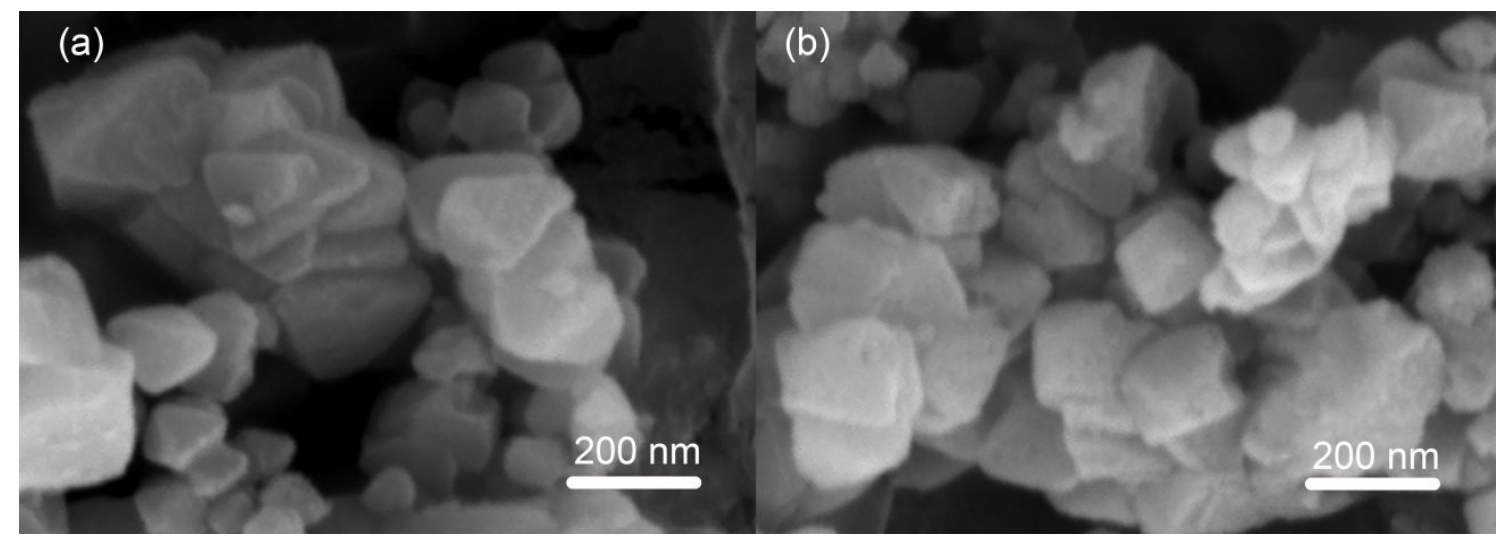

Figure S1. SEM images of (a) UiO-66- $\mathrm{NH}_{2}$ and (b) UiO-66-F.

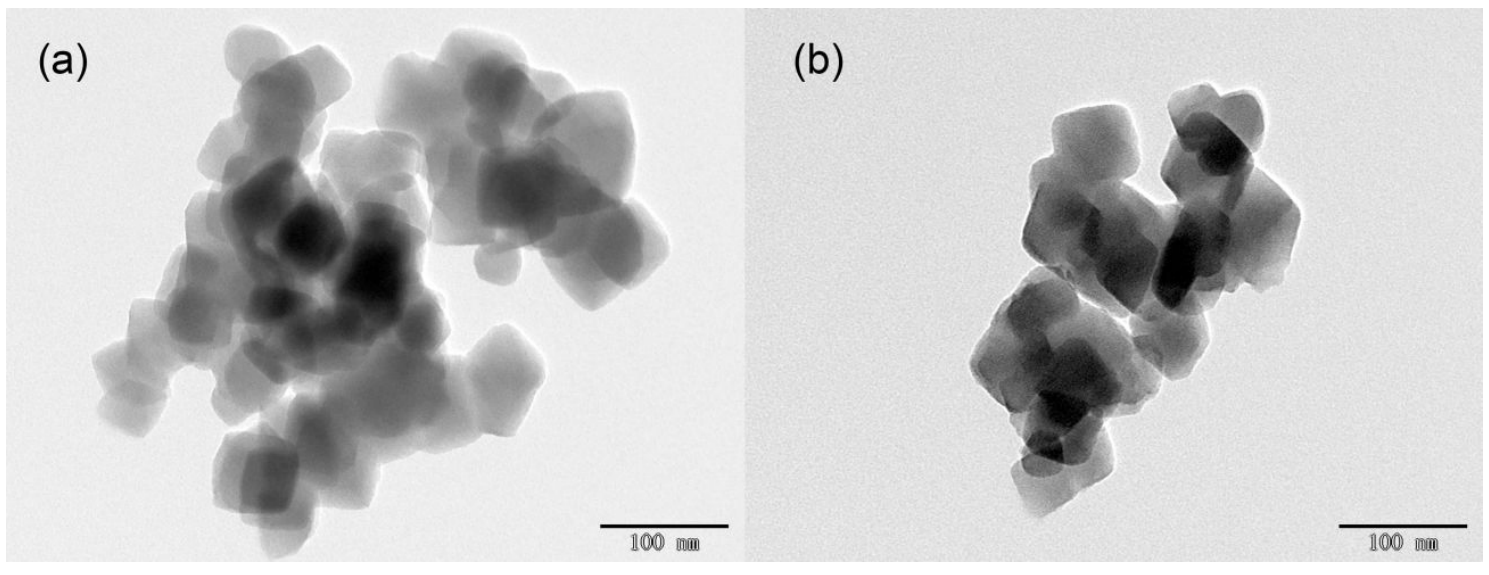

Figure S2. TEM images of (a) $\mathrm{UiO}-66-\mathrm{NH}_{2}$ and (b) $\mathrm{UiO}-66-\mathrm{F}$. 


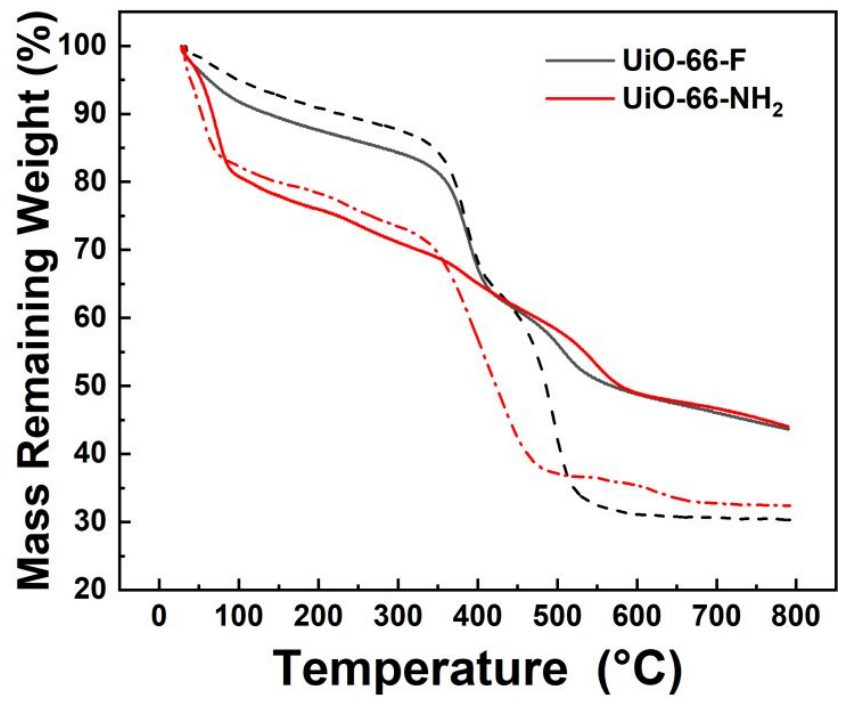

Figure S3. TGA curves of UiO-66- $\mathrm{NH}_{2}$ (red) and UiO-66-F (black) under nitrogen (solid) and air (dash) condition, respectively.

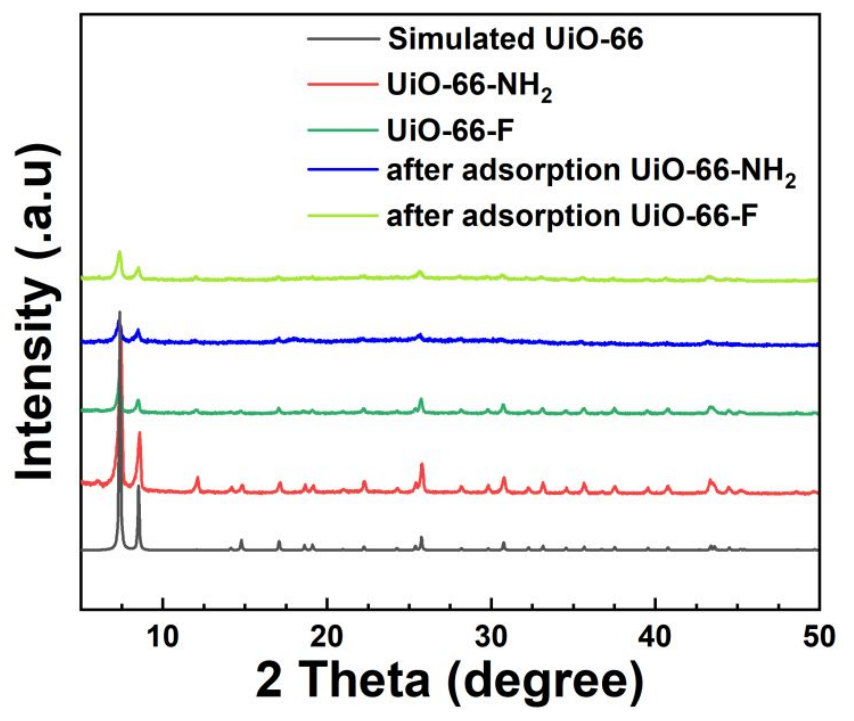

Figure S4. The PXRD patterns of UiO-66-F and UiO-66- $\mathrm{NH}_{2}$ after the adsorption of CBZ. 


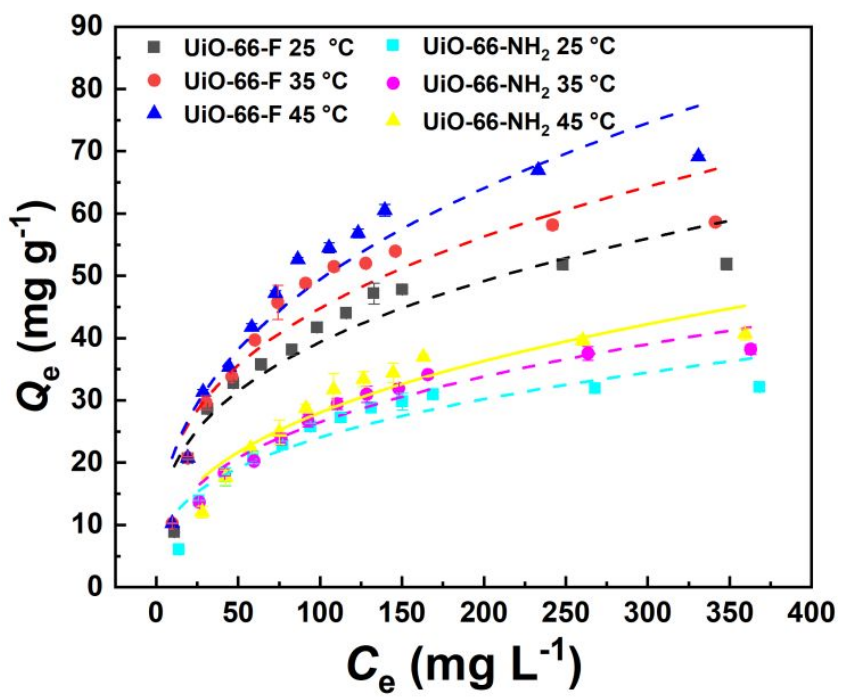

Figure S5. Adsorption isotherms for $\mathrm{CBZ}$ over UiO-66- $\mathrm{NH}_{2}$ and UiO-66-F. (Curves are the results of the fits performed using the Freundlich model.)

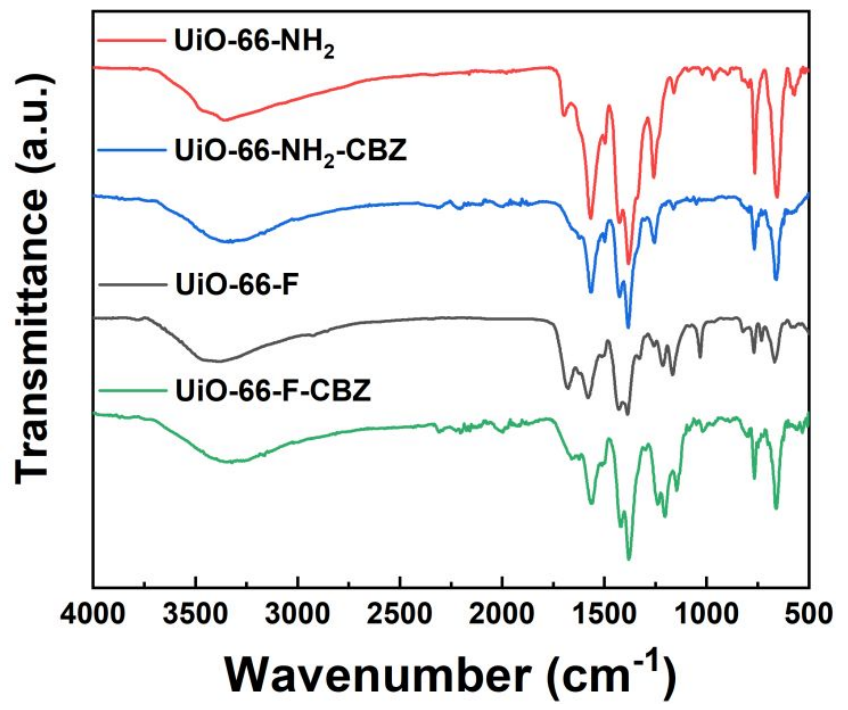

Figure S6. FTIR curves of UiO-66-NH${ }_{2}, \mathrm{UiO}-66-\mathrm{F}, \mathrm{UiO}-66-\mathrm{NH}_{2}-\mathrm{CBZ}$, and UiO-66-F-CBZ. 


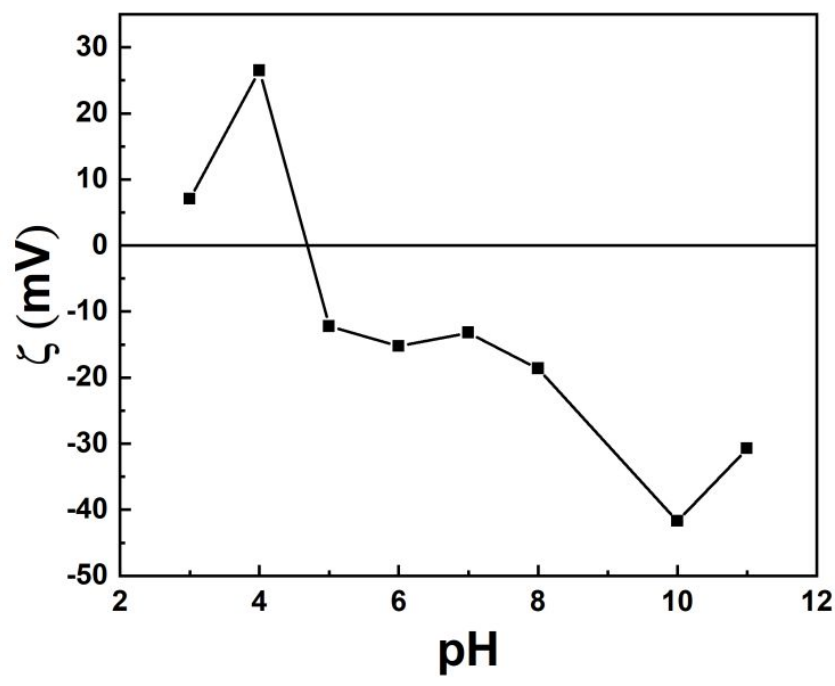

Figure S7. The zeta potential of UiO-66-F at different $\mathrm{pH}$.

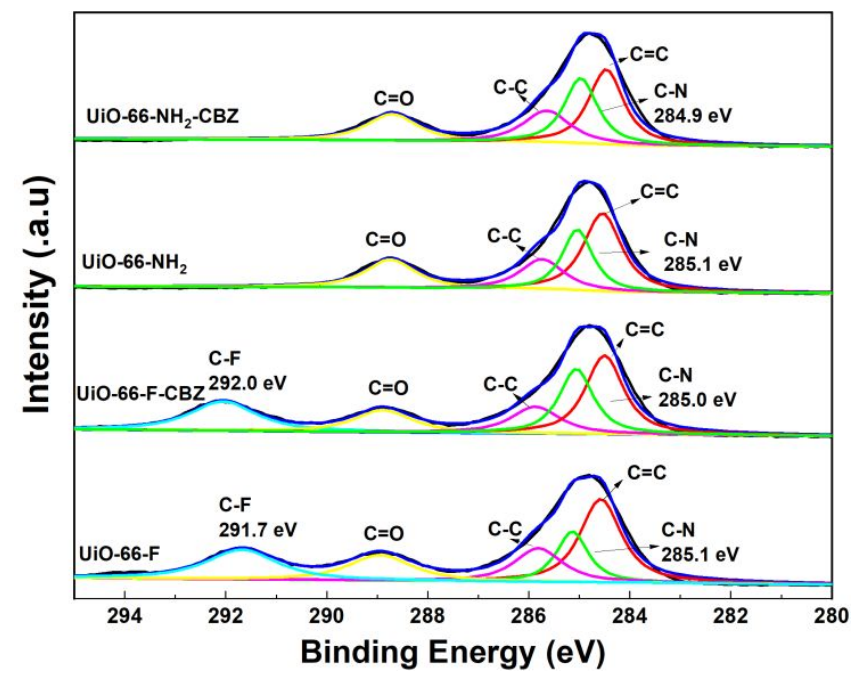

Figure S8. The $\mathrm{C} 1 \mathrm{~s}$ spectra of $\mathrm{UiO}-66-\mathrm{NH}_{2}, \mathrm{UiO}-66-\mathrm{NH}_{2}-\mathrm{CNZ}$, UiO-66-F, and UiO-66-F-CBZ.

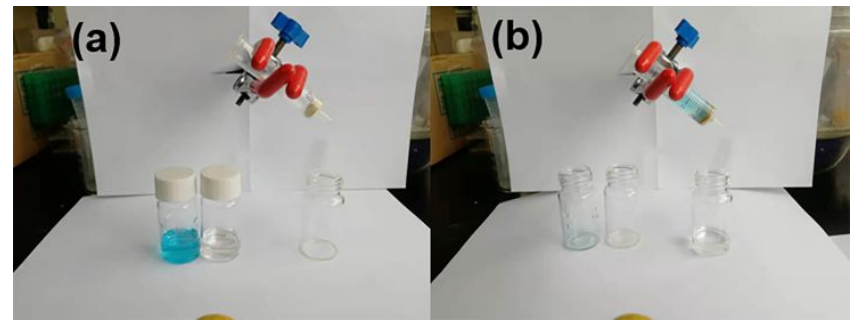

Figure S9. The images of (a) before (b) after separate water/dichloromethane. 
Table S1. Kinetic parameters of CBZ adsorption onto UiO-66- $\mathrm{NH}_{2}$ and UiO-66-F nanoadsorbent.

\begin{tabular}{|c|c|c|c|c|c|c|}
\hline \multirow{2}{*}{ Sample } & \multicolumn{3}{|c|}{ Pseudo-first-order kinetics } & \multicolumn{3}{|c|}{ Pseudo-second-order kinetics } \\
\hline & $\overline{k_{1}\left(\min ^{-1}\right)}$ & $Q_{\mathrm{e}, \mathrm{cal}}\left(\mathrm{mg} \mathrm{g}^{-1}\right)$ & $R^{2}$ & $k_{2}\left(\mathrm{~g}(\mathrm{mg} \min )^{-1}\right)$ & $Q_{\mathrm{e}, \mathrm{cal}}\left(\mathrm{mg} \mathrm{g}^{-1}\right)$ & $R^{2}$ \\
\hline $\mathrm{UiO}-66-\mathrm{NH}_{2}$ & $6.63 \times 10^{-3}$ & 3.88 & 0.969 & $8.89 \times 10^{-3}$ & 15.63 & 0.999 \\
\hline $\mathrm{UiO}-66-\mathrm{F}$ & $3.10 \times 10^{-3}$ & 33.99 & 0.891 & $3.03 \times 10^{-4}$ & 29.34 & 0.999 \\
\hline
\end{tabular}

\section{Reference}

[S1] Hintz, H.; Wuttke, S. Solvent-free and time efficient post-synthetic modification of amino-tagged metal-organic frameworks with carboxylic acid derivatives. Chem. Mater. 2014, 26, 6722-6728. 